\title{
Research on the geometric errors identification of rotary axes on the five-axis machine tool
}

\author{
FAN Jinwei ${ }^{1, a}$,TAO Haohao ${ }^{1, b}$, LV Qi $^{1}$ and LIU Kaikai ${ }^{1}$ \\ ${ }^{1}$ Mechanical and Electrical Engineering College,Beijing University of Technology,Beijing \\ b872781344@qq.com
}

Keywords: five-axis CNC machine tool; double ball-bar; error identification; multi-body system; rotary axis

\begin{abstract}
Aims at AOCMT type five-axis ultra-precision CNC machine tool, the rotary axis geometric errors model is established by applying the homogeneous coordinate transformation matrix, a kind of errors measurement and identification method is put forward. It takes A axis as an example, using the function of RTCP to control a rotary axis and two translational axes synchronous movement. Different height and positions are designed, and the modes of the measurement are the axial, radial and tangential. The length variations are acquired, and the relational expressions of deviations and length variations are derived according to the errors model. Eight deviations related to A axis can be identified at the same time. The method is available through the simulation.
\end{abstract}

\section{Introduction}

With the progress of science and technology, mechanical manufacturing industry has been booming, the shape of the complex, high precision parts demand continues to increase. Five axis NC machine tool is widely used in automobile, mould and ship industry because of its superiority in machining complex curved surface parts. Compared to the traditional three axis machine tool, The five axis machine tool has many advantages such as high machining efficiency, high material removal rate and good surface quality. But its machining accuracy is not as good as three axis machine tool, The main reason lies in the lack of precision calibration and compensation methods for the two axes of rotation ${ }^{[1]}$.It is found that the geometric errors and thermal errors of the machine tools are the main errors in the machining process. When the temperature change is not big, the geometric error is the main part of the error of the machine. It is less affected by the environment, easy to make the error compensation and control. It is one of the main research directions to improve the machining precision ${ }^{[2]}$.

Because of the influence of the error elements of motion axes on the machining accuracy of the five axis machine tool, the detection and error identification of motion axes is an important part of improving the accuracy.Especially, there is no uniform standard for the measurement of the rotating

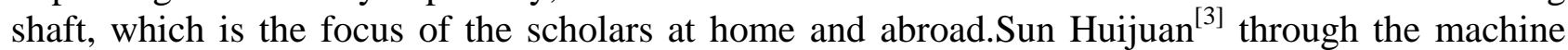
linear axes linkage mode, circular error test was carried out using ballbar, puts forward a comprehensive evaluation method for geometric error and servo error, and its accuracy is verified by experiment.Wang $\mathrm{Min}^{[4]}$ designed a measuring method by using the cue, and the simulation analysis shows that the method can accurately identify the 8 static errors of the machine tool rotary table.Hong $\mathrm{Cefu}^{[5]}$ consider the dynamic error of location point, establish the motion model of the machine tool, through the simulation processing cone and the use of $\mathrm{R}$ testing equipment, to identify the key parameters of the machine. Throughout the domestic and foreign scholars, most of the research is to consider the static error or location point related dynamic error which is independent of the location point. The research on the combination of the two is few.In this paper, we take multi-body system kinematics for basic theory,and use the AOMCT type machine tool as the research object,Two kinds of errors are considered simultaneously, and the method of error measurement and identification is designed by using the ball cue instrument.The method is designed for different measurement models, which are derived from the mathematical expression of the 
length variation and the error parameters. The length variation of the acquisition rod can identify the 8 errors of the A axis, which is fast and accurate ${ }^{[6]}$.

\section{Error parameters of AOCMT type machine tool}

The research object of this paper is the AOCMT type five axis ultra precision machine tool.Its machine tool structure is shown in Figure 1, For this machine, according to the motion characteristics of its components, it can be known that there are 8 errors in each rotation axis motion of the machine.A axis rotation to produce 3 line displacement error $\delta_{x}(\alpha) 、 \delta_{y}(\alpha) 、 \delta_{z}(\alpha), 3$ angle displacement error $\varepsilon_{x}(\alpha) 、 \varepsilon_{y}(\alpha) 、 \varepsilon_{z}(\alpha)$, As shown in Figure 2.At the same time, $\mathrm{A}$ axis and $\mathrm{X}$ axis in practice can not be completely parallel, so it exists and $\mathrm{Y}$ axis and $\mathrm{Z}$ axis Perpendicularity Error $\varepsilon_{A z} 、 \varepsilon_{A y}$, As shown in Figure 3.

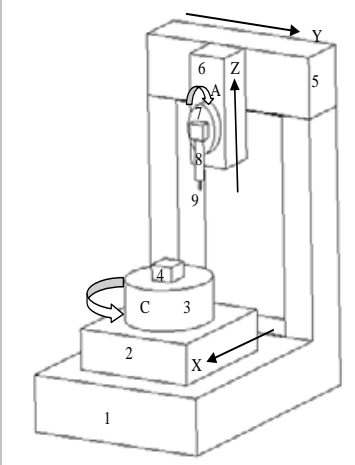

Fig 1AOCMT machine tool structure

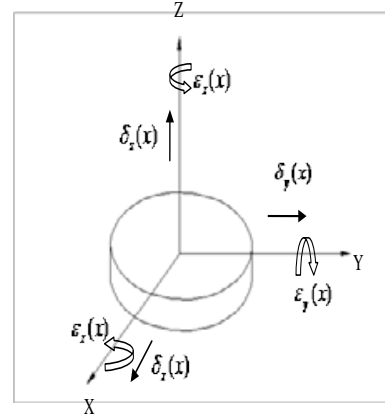

Fig $2 \mathrm{X}$ axis six dynamic errors Perpendicularity Error

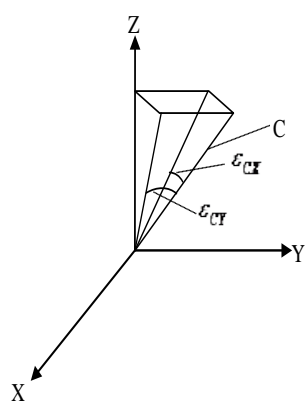

Fig $3 \mathrm{C}$ axis two

\section{Geometric error measurement and identification of $A$ axis}

Measuring instruments used in this paper is the Renishaw company 's QC10 ballbar.Rod instrument can quickly, economically and conveniently measure and evaluate the dynamic accuracy of CNC machine tools, the early application of three axis machine tools, in recent years, the domestic and foreign scholars have been applied to the detection of five axis machine tools.

In front of the analysis we can know the influence of A axis motion accuracy of a total of 8 error parameters, the ball bar measurement model were axial, radial, tangential, as shown in Figure 4.The coordinate system is established with the $\mathrm{A}$ axis turning center as the origin, $R_{a}$ is a distance from $\mathrm{A}$ turntable table to $\mathrm{M}$ sphere, $h_{a}$ is vertical height from $\mathrm{M}$ sphere to $\mathrm{X}$ coordinate axis, Rod length is L,The RTCP function of the machine tool can realize multi axis synchronous motion, and the output signal of the sensor can be read out by the length change of the rod.

As shown in Figure 4, M sphere in homogeneous coordinates is $\left(\begin{array}{llll}R_{a} & 0 & h_{a} & 1\end{array}\right)^{T}$, When the A axis turn $\alpha$ angle, According to the theory of multi body system, $\mathrm{M}$ ball in the position of the homogeneous coordinate system are:

$$
\begin{aligned}
& P_{t}=T_{1} \cdot T_{2} \cdot T_{3} \cdot\left[\begin{array}{llll}
R_{a} & 0 & h_{a} & 1
\end{array}\right]^{T}=\left[\begin{array}{llll}
P_{t x} & P_{t y} & P_{t z} & 1
\end{array}\right]^{T} \\
& T_{1}=\left[\begin{array}{cccc}
1 & -\varepsilon_{A y} & \varepsilon_{A z} & 0 \\
\varepsilon_{A y} & 1 & 0 & 0 \\
-\varepsilon_{A z} & 0 & 1 & 0 \\
0 & 0 & 0 & 1
\end{array}\right]
\end{aligned}
$$




$$
\begin{aligned}
T_{2} & =\left[\begin{array}{cccc}
1 & 0 & 0 & 0 \\
0 & \cos \alpha & -\sin \alpha & 0 \\
0 & \sin \alpha & -\cos \alpha & 0 \\
0 & 0 & 0 & 1
\end{array}\right] \\
T_{3} & =\left[\begin{array}{cccc}
1 & -\varepsilon_{z}(\alpha) & \varepsilon_{y}(\alpha) & \delta_{x}(\alpha) \\
\varepsilon_{z}(\alpha) & 1 & \varepsilon_{x}(\alpha) & \delta_{y}(\alpha) \\
-\varepsilon_{y}(\alpha) & -\varepsilon_{x}(\alpha) & 1 & \delta_{z}(\alpha) \\
0 & 0 & 0 & 1
\end{array}\right]
\end{aligned}
$$

After simplification:

$$
\begin{aligned}
& P_{t x}=\delta_{x}(\alpha)+R_{a}+h_{a}\left[\varepsilon_{y}(\alpha)+\varepsilon_{A y} \sin \alpha+\varepsilon_{A z} \cos \alpha\right] \\
& P_{t y}=\delta_{y}(\alpha) \cos \alpha-\varepsilon_{z}(\alpha) \sin \alpha+R_{a}\left[\varepsilon_{A y}+\varepsilon_{y}(\alpha) \sin \alpha+\varepsilon_{z}(\alpha) \cos \alpha\right]-h_{a}\left[\sin \alpha+\varepsilon_{x}(\alpha) \cos \alpha\right] \\
& P_{t z}=\varepsilon_{z}(\alpha) \cos \alpha+\varepsilon_{y}(\alpha) \sin \alpha-R_{a}\left[\varepsilon_{A z}+\varepsilon_{y}(\alpha) \cos \alpha-\varepsilon_{z}(\alpha) \sin \alpha\right]-h_{a}\left[\cos \alpha+\varepsilon_{x}(\alpha) \sin \alpha\right]
\end{aligned}
$$

As shown in Figure 4a,The homogeneous coordinates of the ball $\mathrm{N}$ in the machine tool coordinate system are $\left(R_{a}+L, 0, h_{a}, 1\right)^{T}$,So we can know that the A axis turns $\Delta L=\sqrt{\left(P_{w X}-P_{t X}\right)^{2}+\left(P_{w y}-P_{t y}\right)^{2}+\left(P_{w Z}-P_{t Z}\right)^{2}}$ angle, The homogeneous coordinates of the ball $\mathrm{N}$ in the coordinate system are:

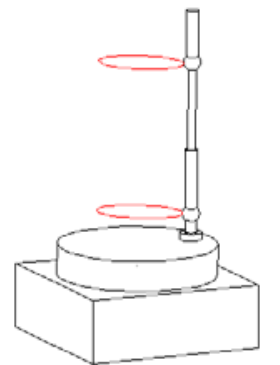

(a) Axial measuremen

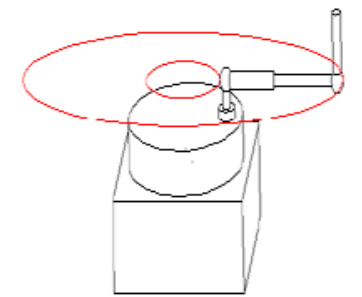

(b) Radial measurement

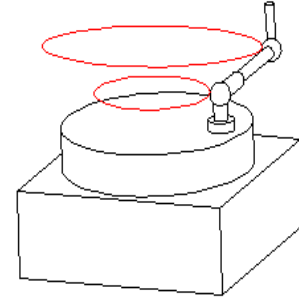

(c) Tangential measurement

Figure4 Three measuring modes of the cue instrument

$$
p_{M_{x}}=\left[\begin{array}{cccc}
1 & 0 & 0 & 0 \\
0 & \cos \alpha & -\sin \alpha & 0 \\
0 & \sin \alpha & \cos \alpha & 0 \\
0 & 0 & 0 & 1
\end{array}\right]\left[\begin{array}{c}
R_{a}+L_{s a} \\
0 \\
h_{a} \\
1
\end{array}\right]=\left[\begin{array}{c}
R_{a}+L_{s a} \\
-h_{a} \sin \alpha \\
h_{a} \cos \alpha \\
1
\end{array}\right]
$$

Similarly, according to figure $4 \mathrm{~b}$ 、 $\mathrm{c}$, we can get the ball $\mathrm{N}$ position of the homogeneous coordinates are $\left(R_{a}, 0, h_{a}, 1\right)^{T} 、\left(R_{a}, L_{t a}, h_{a}, 1\right)^{T}$.The homogeneous coordinates of the $\mathrm{N}$ in the coordinate system are further available.

$$
\begin{aligned}
& p_{M_{r}}=\left[\begin{array}{cccc}
1 & 0 & 0 & 0 \\
0 & \cos \alpha & -\sin \alpha & 0 \\
0 & \sin \alpha & \cos \alpha & 0 \\
0 & 0 & 0 & 1
\end{array}\right]\left[\begin{array}{c}
R_{a} \\
0 \\
h_{a}-L_{r a} \\
1
\end{array}\right]=\left[\begin{array}{c}
R_{a} \\
\left(L_{r a}-R_{a}\right) \sin \alpha \\
\left(h_{a}-L_{r a}\right) \cos \alpha \\
1
\end{array}\right] \\
& p_{M_{t}}=\left[\begin{array}{cccc}
1 & 0 & 0 & 0 \\
0 & \cos \alpha & -\sin \alpha & 0 \\
0 & \sin \alpha & \cos \alpha & 0 \\
0 & 0 & 0 & 1
\end{array}\right]\left[\begin{array}{c}
R_{a} \\
L_{a} \\
h_{a} \\
1
\end{array}\right]=\left[\begin{array}{c}
L_{t a} \cos \alpha-h_{a} \sin \alpha \\
L_{t a} \sin \alpha+h_{a} \cos \alpha \\
1
\end{array}\right]
\end{aligned}
$$


The formula for the length variation of the rod can be got.:

$$
\Delta L=\sqrt{\left(P_{w X}-P_{t x}\right)^{2}+\left(P_{w y}-P_{t y}\right)^{2}+\left(P_{w Z}-P_{t z}\right)^{2}}
$$

In the formula $P_{w X} 、 P_{w y} 、 P_{w z}$ is the coordinate of the ball $\mathrm{N}$ in the coordinate system.we put the coordinate values from the formula (8) (9) (10) into equation (11), can be simplified:

$$
\begin{aligned}
& \Delta L_{s a}=-\delta_{x}(\alpha)-h_{a}\left[\varepsilon_{y}(\alpha)+\varepsilon_{A y} \sin \alpha+\varepsilon_{A z} \cos \alpha\right] \\
& \Delta L_{r a}=\delta_{z}(\alpha)-R_{a}\left[\varepsilon_{y}(\alpha)+\varepsilon_{A y} \sin \alpha+\varepsilon_{A z} \cos \alpha\right] \\
& \left.\Delta L_{t a}=-\delta_{y}(\alpha)+R_{a}\left[\varepsilon_{A z} \sin \alpha-\varepsilon_{A y} \cos \alpha-\varepsilon_{z}(\alpha)\right]+h_{a} \varepsilon_{x}(\alpha)\right]
\end{aligned}
$$

When the cue is in the initial position,the angular $\alpha=0$,In this we can make the following assumptions, The initial position error is zero.By formula (11) and formula (14),we use the height $h_{a 1}$ and the distance $R_{a 1}$ to measured length changes were $\Delta L_{s a}$ and $\Delta L_{t a}$.From the above assumptions we can get this result :

$$
\varepsilon_{A z}=\frac{-\Delta L_{s a 1}}{h_{a 1}} \quad \varepsilon_{A y}=\frac{-\Delta L_{t a 1}}{R_{a 1}}
$$

In the formula (12), take different heights $h_{a 1} 、 h_{a 2}$ to measure length variation of the $\operatorname{rod} \Delta L_{s a 1}$ 、 $\Delta L_{s a 2}$.In the formula (13), take different distance $R_{a 1} 、 R_{a 2}$ to measure ength variation of the $\operatorname{rod} \Delta L_{r a 1} 、 \Delta L_{r a 2}$,Simultaneous formula (12) (13), we can get:

$$
\begin{aligned}
& \delta_{x}(\alpha)=\frac{\Delta L_{r a 1}-\Delta L_{r a 2}}{R_{a 1}-R_{a 2}} h_{a 1}-\Delta L_{s 1} \\
& \varepsilon_{y}(\alpha)=\frac{\Delta L_{s a 1}-\Delta L_{s a 2}}{h_{a 2}-h_{a 1}}+\frac{\Delta L_{t a 1}}{R_{a 1}} \sin \alpha+\frac{\Delta L_{s a 1}}{h_{a 1}} \cos \alpha \\
& \delta_{z}(\alpha)=\Delta L_{r a 1}-R_{a 1} \frac{\Delta L_{r a 1}-\Delta L_{r a 2}}{R_{a 1}-R_{a 2}}
\end{aligned}
$$

In the formula (14), take different heights $h_{a 1} 、 h_{a 2}$ and same distance $R_{a 1}$ to measure ength variation of the $\operatorname{rod} \Delta L_{t a 1} 、 \Delta L_{t a 2}$, we can get:

$$
\varepsilon_{x}(\alpha)=\frac{\Delta L_{t a 1}-\Delta L_{t a 2}}{h_{a 1}-h_{a 2}}
$$

In the formula (14), take different distance $R_{a 1} 、 R_{a 2}$ and same heights $h_{a 1}$ to measure length variation of the $\operatorname{rod} \Delta L_{t a 1} 、 \Delta L_{t a 3}$,

$$
\begin{aligned}
& \varepsilon_{z}(\alpha)=\frac{\Delta L_{t a 1}}{R_{a 1}} \cos \alpha-\frac{\Delta L_{s a 1}}{h_{a 1}} \sin \alpha-\frac{\Delta L_{t a 1}-\Delta L_{t a 2}}{R_{a 1}-R_{a 2}} \\
& \delta_{y}(\alpha)=R_{a 1} \frac{\Delta L_{t a 1}-\Delta L_{t a 3}}{R_{a 1}-R_{a 2}}+h_{a 1} \frac{\Delta L_{t a 1}-\Delta L_{t a 2}}{h_{a 1}-h_{a 2}}-\Delta L_{t a 1}
\end{aligned}
$$

In this way, we can identify all the eight errors associated with the A axis.。

\section{Simulation verification}

Assignment of the A axis eight error parameters, in order to ensure the accuracy, a constant of 2 to 6 error, dynamic error, error data acquisition experience other machine is identified, the average of each data error of the corresponding assignment, and determine the corresponding mean angle, we take rod length $\mathrm{L}=100 \mathrm{~mm}$,height ha $=200 \mathrm{~mm}$,distance $\mathrm{Ra}=100 \mathrm{~mm}$. we take the error and angle values into type (12), (13), (14),Calculation of the corresponding rod length change,Then the 
change amount of generation (15) (21), can get the error of the identification value, as shown in Table 1.As can be seen from the table, the difference of the identification results of the errors in the allowable range, so the identification method proposed in this paper is accurate.

\section{Conclusion}

In this paper, based on the AOCMT five axis machine tool, based on the multi-body system kinematics to establish the error model, the use of RTCP function to control the motion of the machine tool, and the use of the ball bar instrument to measure its A axis, 1 error identification methods are proposed.The method can separate the static error and dynamic error of the A axis by 7 measurements, and the method is validated by simulation. The method is accurate and efficient.Compared with the method which only considers the static error and the dynamic error, the method is very novel and practical, and it has important significance for the error compensation of five axis machine tool.

\section{Reference}

[1] Hsu Y Y,Wang S S. A new compensation method for geometry errors of a five-axis machine tools [J] . International Journal of Machine Tools\& Manufacture ,2007 47;352-360.

[2] LIU Jianjun.Research on geometric error identification and compensation technology of multi axis NC machine tools[D].Chengdu:Southwest Jiao Tong University, 2012.

[3] SUN Huijuan, YIN Guofu, FANG Hui.Research on comprehensive error modeling and evaluation method of five axis NC machine tools[J].Journal of Sichuan University:Engineering Science Edition, 2012,44 (6) :197-202.

[4] WANG Min, HU Jianzhong, ZHOU Tao.Motion error modeling and testing technology of five axis NC machine tools[J].Journal of Beijing University of Technology, 2010,36(4);433-439.

[5] Cefu Hong, Soichi Ibaraki , Atsushi Matsubara.Influence of position-de-pendent geometric of rotary axes on a machining test of cone frus-tum by five-axis machine tools[J].Precision Engineering,2011(35);1 11.

[6] Tsutumi M , Saito A ,Identification and compensation of systematic devia-lions particular to 5-axis machining centers[J].International Journal of Machine Tools\&Manufacture,2003 (43):771-780. 